Check for updates

Cite this: RSC Adv., 2017, 7, 20147

\title{
Thiol-ene click reaction on cellulose sponge and its application for oil/water separation
}

\author{
Zhenzhen Wu, Yingzhan Li, Linping Zhang, ${ }^{*}$ Yi Zhong, Hong Xu, Zhiping Mao, \\ Bijia Wang and Xiaofeng Sui (D)*
}

Thiol-ene click reaction was employed to synthesize a flexible hydrophilic cellulose sponge. The reactive vinyl group was introduced by silanization treatment with vinyl-trimethoxysilicane (VTMO), and the resulting silylated cellulose sponge (SCS) can be subsequently functionalized with various thiol-containing compounds such as 3-mercaptopropionic acid, via temperature induced thiol-ene reactions. The hydrophilic cellulose sponge thus prepared displayed an excellent mechanical strength of $70 \mathrm{KPa}$ at $80 \%$ compression strain. The prepared sponge features hydrophilic and underwater oleophobic properties and was used in gravity-driven removal of oil from oil-in-water emulsions and oil/water mixtures with a high separation efficiency. The separation efficiency remained $100 \%$ after 10 cycles for an oil/water mixture and $95 \%$ after 6 cycles for an oilin-water emulsion.

Received 20th January 2017

Accepted 23rd March 2017

DOI: $10.1039 / \mathrm{c} 7 \mathrm{ra00847c}$

rsc.li/rsc-advances

further discussed the recyclability for oil/water emulsion sepa-

\section{Introduction}

Water pollution from oil spillage and industrial discharge of organic solvents has become more and more serious, making oil/ water separation of worldwide urgency. Adsorption-based oil/ waster separation methods are attractive for being energyefficient. Inorganic sorbents including silica aerogels, ${ }^{1,2}$ carbon aerogels, ${ }^{3,4}$ graphene aerogels ${ }^{5,6}$ and organic synthetic polymers such as polystyrene, ${ }^{7}$ polypropylene,${ }^{8}$ polyurethane foam, ${ }^{9}$ and melamine sponges ${ }^{10}$ have been demonstrated to function as oil removal materials that adsorb oil from water. However, the widespread utilization of these sorbents are hindered by their complicated fabrication procedures and lack of biodegradability. Thus, there was great interest in developing eco-friendly materials as effective oil/water separation mediums.

Cellulose sponge, a porous material, has been developed for oil/water separation particularly via absorption due to their fascinating characters, such as sustainable origins, low-cost, and biocompatibility. ${ }^{11-16}$ However, these absorption materials easily fouled by oil due to theirs oleophilicity, which can reduce the absorption capacity. ${ }^{17-19}$ Therefore, it's necessary to explore hydrophilic porous cellulose materials for oil/water separation via filtration through chemical modifications. Batchelor et al. ${ }^{20}$ used polyamideamine-epichlorohydrin in preparing a hydrophilic cellulose aerogel, which they successfully applied in oil/ water separation. Hu et al. ${ }^{21}$ prepared a hydrophilic cellulosebased filtering material crosslinked by citric acid for oil/water separation. However, neither of the above mentioned work

Key Lab of Science and Technology of Eco-textile, Ministry of Education, Donghua University, Shanghai 201620, P. R. China. E-mail: suixf@dhu.edu.cn; zhanglp@ dhu.edu.cn; Fax: +8621 67792707; Tel: +862167792605; +862167792707 ration of the materials.

The current study involves using thiol-ene click reaction to treat cellulose sponge with vinyl-trimethoxysilicane (VTMO), reintroduces hydrophilicity into the rather hydrophobic silanecrosslinked cellulose sponge. Thiol-ene click reactions ${ }^{22,23}$ are widely employed to introduce functional groups, such as $-\mathrm{OH}$, $-\mathrm{COOH}$, and $-\mathrm{NH}_{2}$ to cellulose. Tingaut et al. ${ }^{24}$ prepared three functional cellulose films using thiol-ene click reactions. Zhang et $a .^{25}$ prepared different $\mathrm{pH}$-responsive nanoparticles from novel ionic cellulose derivatives made by thiol-ene click reactions. Levkin et al. ${ }^{26}$ prepared functional nanocellulose films using photo-induced thiol-ene reactions.

Crosslinked cellulose sponge was prepared by treating cellulose fibrils with vinyl-trimethoxysilicane (VTMO), where OSi crosslinking and the introduction of the reactive vinyl groups were achieved simultaneously. The resulted silylated cellulose

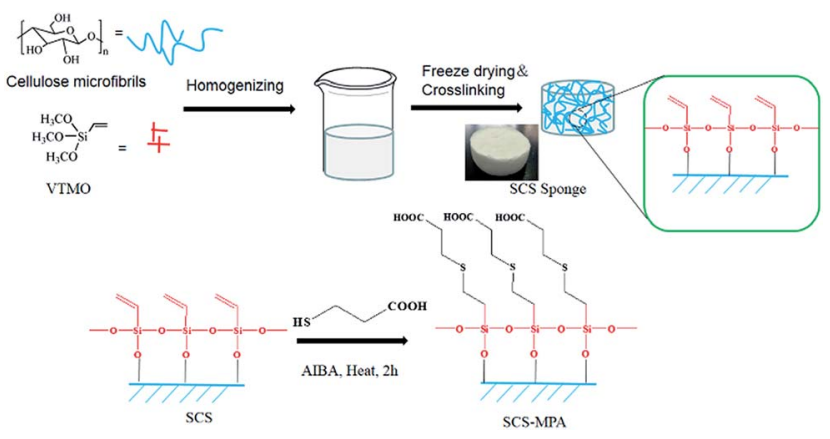

Scheme 1 Scheme showing the preparation of thiol-ene modified silylate cellulose sponge (SCS-MPA) from cellulose microfibrils. 
sponge (SCS) was then reacted with 3-mercaptopropionic acid (MPA) and to get grafted with the hydrophilic carboxylate moieties via thiol-ene reaction (Scheme 1). The surface of the modified SCS sponge showed underwater oleophobicity. The sponge was demonstrated to rapidly separate oil from the oil/ water mixtures, and also exhibited decent performance and excellent recyclability in separating oil-in-water emulsions.

\section{Experimental section}

\subsection{Materials}

Cellulose microfibrils suspension (solid content 1.4\%) was purchased from Tianjin Haojia Cellulose CO., Ltd, China. VTMO was supplied by Zhejiang Feidian Chemical Co., Ltd. 2,2' Azobis[2-methylpropionamidine]dihydrochloride (AIBA), 3mercaptopropionic acid (MPA) was obtained from Energy Chemical. Hydrochloric acid ( $\mathrm{HCl})(37 \%)$ was obtained from Pinghu Chemical Reagent, China. $n$-Hexane was purchased from Changshu Hongsheng Fine Chemical Co., Ltd. Acetone, ethanol was provided by ShangHai Yunli Economic and Trade Co., Ltd. Disperse blue SE-5R was produced by Zhejiang JiHua Group. Deionized water was used in all experiments.

\subsection{Synthesis of silylated cellulose sponge (SCS)}

$140 \mathrm{mg}$ VTMO was added dropwise into $10 \mathrm{~g}$ cellulose microfibrils suspension. The suspension was stirred for $2 \mathrm{~h}$ at room temperature under acidic conditions $(\mathrm{pH}=4)$. The mixture was frozen in liquid nitrogen and freeze-dried in freeze-dryer (DGJT10, Beijing Songyuan Huaxing Technology Development Co., Ltd.) for $48 \mathrm{~h}$. Finally, dry sponge was cured at $120^{\circ} \mathrm{C}$ for $10 \mathrm{~min}$ to promote the cross-linking between cellulose and VTMO.

\subsection{Preparation of SCS-MPA sponge via thiol-ene click reaction}

$79.5 \mathrm{mg}$ AIBA (0.293 mmol) was dissolved in water $(5 \mathrm{~mL}) .10 \mathrm{~mL}$ ethanol and $1 \mathrm{~g}$ (9.42 mmol) MPA were added to the solution successively. The mixture was shaken in a vortex mixer. Upon thorough mixing, $200 \mathrm{mg}$ SCS was added, the mixture was heated to $60{ }^{\circ} \mathrm{C}$ and maintained for $2 \mathrm{~h}$. The sample was rinsed with water and ethanol sufficiently to remove the unreacted materials. Finally, the sample was dried at $80{ }^{\circ} \mathrm{C}$ for $4 \mathrm{~h}$ and stored in a desiccator.

\subsection{Characterization}

2.4.1. Analysis of structure and morphology. The chemical constitution of the original cellulose sponge (CS), SCS and SCSMPA sponges were studied using Fourier transform infrared (FT-IR) spectrometry (PerkinElmer Spectrum Two, American). All spectra were recorded between 4000 and $600 \mathrm{~cm}^{-1}$ with a resolution of $4 \mathrm{~cm}^{-1}$ scans.

The morphologies of the samples were investigated using scanning electron microscopy (SEM, TM-1000, HITACHI, Japan). The $\mathrm{N}_{2}$ adsorption-desorption isotherms and BET surface area were characterized by a TriStar II 3020M analyzer (Micrometrics instrument corp.).
The dynamic water contact angle (WCA) and oil contact angle on underwater (OCA) were collected with a DSA30 contact angle analyzer (Kruss, German). Optical microscopy images of the emulsions and filtrates were captured by a Nikon $80 \mathrm{i}$ optical microscope. The amounts of oil in the emulsion and the filtrate were determined by UV-Vis spectroscopy (UV-1800, Shimadzu corp., Japan).

2.4.2. Mechanical testings. The compression tests were performed on a HY-940F Universal Testing System (Shanghai). The samples were placed between the testing plates and compressed at a speed of $2 \mathrm{~mm} \mathrm{~min}^{-1}$ with the maximum compression strain $(\varepsilon)$ set to $80 \%$. Young's modulus and compression strength were obtained from the stress curves. The thickness recovery was calculated according to the following formula: ${ }^{14}$

$$
S \%=100-\varepsilon_{\text {final }}
$$

where $\varepsilon_{\text {final }}$ was the residual strain and could be measured at the final position, where no stress was applied. An average of three replicates were used for each sample.

2.4.3. Evaluation of the density and porosity of SCS sponge. The porosity was calculated using the following equation:

$$
\text { Porosity }(\%)=\left(1-\frac{\rho_{\mathrm{b}}}{\rho_{\mathrm{s}}}\right) \times 100 \%
$$

Where $\rho_{\mathrm{b}}$ was the bulk density and $\rho_{\mathrm{s}}$ was the skeletal density of the SCS sponge. $\rho_{\mathrm{b}}$ was calculated from the weight and bulk volume of the sponge. Considering that the SCS material could be regarded as a composite of cellulose and VTMO, $\rho_{\mathrm{s}}$ was calculated according to eqn (3):

$$
\begin{aligned}
\rho_{\mathrm{s}} & =\frac{1}{\frac{W_{\text {cellulose }}}{\rho_{\text {cellulose }}}+\frac{W_{\mathrm{VTMO}}}{\rho_{\mathrm{VTMO}}}} \\
& =\frac{\rho_{\mathrm{VTMO}} \rho_{\text {cellulose }}}{W_{\text {cellulose }} \rho_{\mathrm{VTMO}}+\left(1-W_{\text {cellulose }}\right) \rho_{\text {cellulose }}}
\end{aligned}
$$

where $W_{\text {cellulose }}$ was the weight fraction of cellulose and $W_{\text {VTMO }}$ was the weight fraction of VTMO in the sponge $\left(W_{\text {cellulose }}+W_{\text {VTMO }}=1\right)$. $\rho_{\text {cellulose }}$ and $\rho_{\text {VTMO }}$ were obtained according to literatures. ${ }^{\mathbf{1 4 2 7 - 2 9}}$

2.4.4. Evaluation of the oil rejection $(R \%)$ of SCS-MPA sponge. The oil/water mixture was prepared by vigorously stirring. To prepare the surfactant-stabilized oil-in-water emulsions, sodium dodecyl-benzenesulfonate $(0.5 \mathrm{~g})$ and $n$-hexane (5 $\mathrm{mL}$ ) was added to $5 \mathrm{~mL}$ of water with intensive mixing. The mixture formed a homogeneous dispersion in about $1 \mathrm{~min}$.

The oil rejection $(R \%)$ was calculated as follow: ${ }^{20}$

$$
R \%=\left(1-\frac{C_{1}}{C_{0}}\right) \times 100
$$

where $C_{0}$ and $C_{1}$ are the concentrations of oil in the emulsion and filtrate, respectively.

\section{Results and discussion}

\subsection{Structure and morphology}

As illustrated in Fig. 1, compared with unmodified CS, the IR spectrum of SCS shows new peaks at $3062 \mathrm{~cm}^{-1}\left(\nu_{\mathrm{as}}=\mathrm{CH}_{2}\right), 3026$ 
$\mathrm{cm}^{-1}\left(\nu_{=\mathrm{CH}}\right), 2969 \mathrm{~cm}^{-1}\left(\nu_{\mathrm{s} \mathrm{CH}_{2}}\right), 1601\left(\nu_{\mathrm{C}=\mathrm{C}}\right), 1408 \mathrm{~cm}^{-1}\left(\delta_{=\mathrm{CH}_{2}}\right.$ and $\delta=\mathrm{CH}$ ), all of which were attribute vibrations of the vinyls on VTMO ${ }^{30,31}$ Moreover, the peak at $1276 \mathrm{~cm}^{-1}\left(\nu_{\mathrm{Si}-\mathrm{C}}\right)$, and between $1180-690 \mathrm{~cm}^{-1}$ were due vibrations of silyl connections $\left(\nu_{\mathrm{Si}-\mathrm{C}}\right.$, $\left.\nu_{\text {as Si-C }}, \nu_{\text {as Si-O-Si }}, \nu_{\text {s Si-O-Si }}\right){ }^{31}$ Upon modification with MPA, the sponge showed a characteristic carbonyl stretching vibration at $1715 \mathrm{~cm}^{-1}\left(\nu_{\mathrm{C}=\mathrm{O}}\right)$, meanwhile the vinyl vibration bands at 3062 , $3026,1600,1408 \mathrm{~cm}^{-1}$ were considerably weakened, indicating the vinyls have transformed to thioether linkages. ${ }^{24}$

The morphology of SCS and SCS-MPA was studied by SEM (Fig. 2). Both SCS and SCS-MPA sponges displayed a porous structure composed of thin sheets interconnected with microfilaments. These thin sheets likely formed from the aggregation of cellulose microfibrils during freeze-drying step, when water within the sponge crystalized. ${ }^{32,33}$ After the thiol-ene chick reaction, the resulted SCS-MPA remained porous. ${ }^{34}$ The porosity of the cellulose sponges was calculated according to eqn (2) and (3), which is a common method to evaluate the porosity of nanocellulose sponge. ${ }^{11,35}$ Results (Table 1 ) showed that regardless of silane modification, the cellulose sponges were highly porous (porosity $\geq 99 \%$ ).

\subsection{Mechanical properties}

The compressive stress-strain curves of the cellulose sponges compressed at $80 \%$ compression strain are presented in Fig. 3a. At $80 \%$ compression strain, the compressive stress of SCS-MPA was about $70 \mathrm{KPa}$. The thickness recovery of unmodified CS was calculated to be $39 \%$, while that of the SCS-MPA was

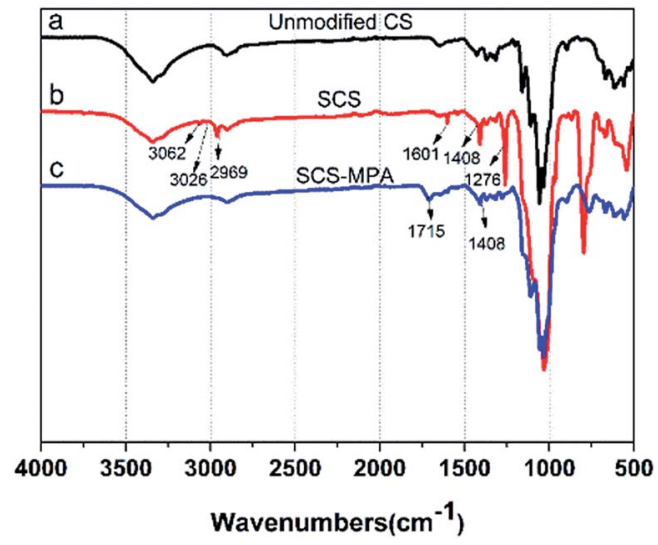

Fig. 1 FTIR spectra of (a) unmodified CS and (b) SCS and (c) its thiolene coupling products SCS-MPA.
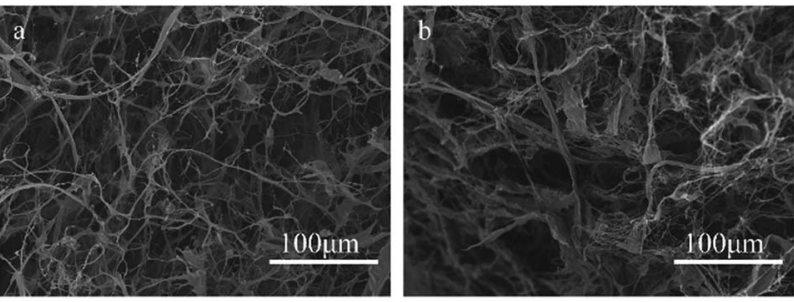

Fig. 2 SEM image of (a) SCS, and (b) SCS-MPA sponge.
Table 1 Density, porosity of unmodified and silylated cellulose sponge

\begin{tabular}{lll}
\hline Sample & Density $/ \mathrm{kg} \mathrm{m}^{-3}$ & Porosity $/ \%$ \\
\hline CS & 10.9 & 99.3 \\
SCS & 12.3 & 99.4
\end{tabular}
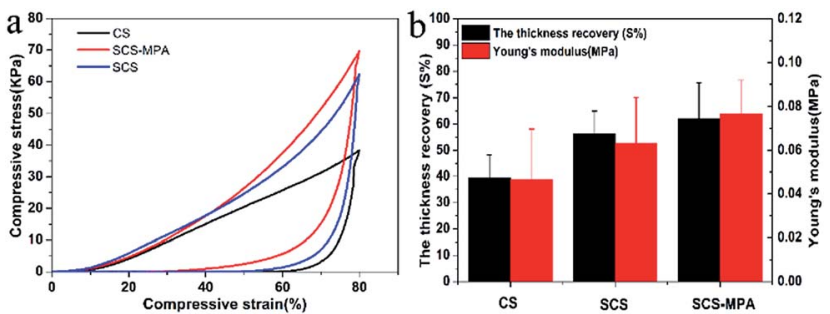

Fig. 3 (a) Compressive stress-strain curves of unmodified CS, SCS and SCS-MPA compressed to $80 \%$ strain, (b) thickness recovery (S\%) and Young's modulus of unmodified CS, SCS and SCS-MPA upon unloading from a compressed state $(\varepsilon=80 \%)$ was visualized by columns.

significantly increased to around $62 \%$ (Fig. $3 \mathrm{~b}$ ). SCS-MPA could recover to its original height in $30 \mathrm{~min}$, while the unmodified CS remained compressed. In addition to being more flexible and resilient, the SCS-MPA sponge also showed higher compressive strength compared with unmodified CS. These observations were in line with the theory that crosslinking enhances the mechanical properties of cellulose sponge..$^{36,37}$

\subsection{Hydrophilic and underwater oleophobic properties}

SCS is highly hydrophobic with water contact angle of $160^{\circ}$. After the thiol-ene reaction, carboxyl groups were grafted onto SCS. ${ }^{38}$ The surface of the sponge changed from hydrophobic to hydrophilic and displayed underwater oleophobicity. Both surfaces of unmodified CS and SCS-MPA sponge were highly hydrophilic as demonstrated in Fig. 4a. However, the CS surface collapsed after absorbing the water-droplets, while the SCS-MPA surface remained intact. The SCS-MPA sponge also showed
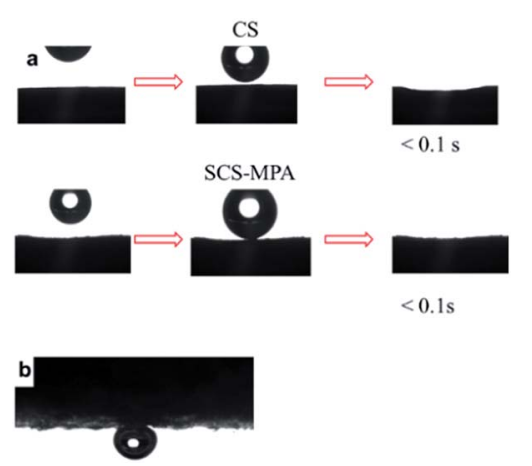

Fig. 4 (a) Photographs of dynamic measurements of water permeation on the surface of CS and SCS-MPA sponge, (b) oil contact angle on the SCS-MPA sponge underwater (contact angle: $147^{\circ}$ ). 
oleophobicity when immersed in water, exhibiting a high oil contact angle of $147^{\circ}$ (Fig. $4 \mathrm{~b}$ )..$^{10}$ Therefore, the present material was used for oil/water separation.

\subsection{Separation capacity and recyclability}

As expected, the wetted cellulose sponge, which showed underwater oleophobic, facilitated the efficient separation of oil/water mixtures. The oil/water mixture separation experiment was performed as depicted in Fig. 5a. The water-removing process was driven by gravity. For oil/water mixture $(50 \mathrm{~mL}$, $1: 1, \mathrm{v} / \mathrm{v}$ ), stratification took place when it was poured into the glass graduated funnel. Water rapidly passed through the materials $(<2 \mathrm{~min})$, while hexane was intercept due to the hydrophilic and underwater oleophobic characteristics of the sponge. Consequently, SCS-MPA has unexpectedly high separation efficiencies for oil/water mixture. The oil rejection, calculated by eqn (4), was studied after every separation experiment and the results are summarized in Fig. 5b. The oil rejection was $100 \%$ for all ten repeated cycles, demonstrating a favorable reusability feature of the SCS-MPA sponge.

The separation efficiency of SCS-MPA was further evaluated using oil-in-water emulsions. Passing through the sponge caused the emulsion to demulsify and the coalesced oil phase was retained above the sponge. ${ }^{39}$ The optical microscopic images of the oil-in-water emulsion and the filtrate were shown in Fig. 6. The filtrate clearly contained no oil droplet, suggesting the SCS-MPA sponge was effective in removing oil from the surfactant-stabilized oil-in-water emulsion. The UV-Vis spectrum was also employed to analyze the filtrate, from which the rejection ratio of oil was determined to be as high as $98.6 \%$
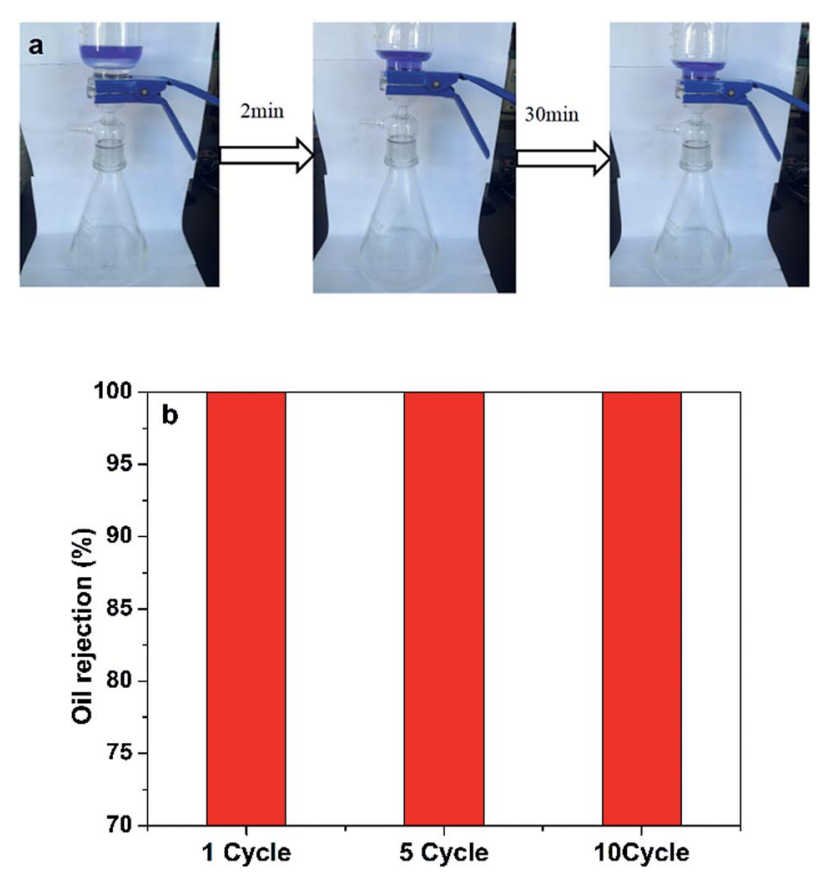

Fig. 5 (a) The photographs of hexane/water mixtures separation process (the disperse blue dye SE-5R was dissolved in the oil phase), (b) efficiency and reusability of the SCS-MPA on separating oil/water mixtures.
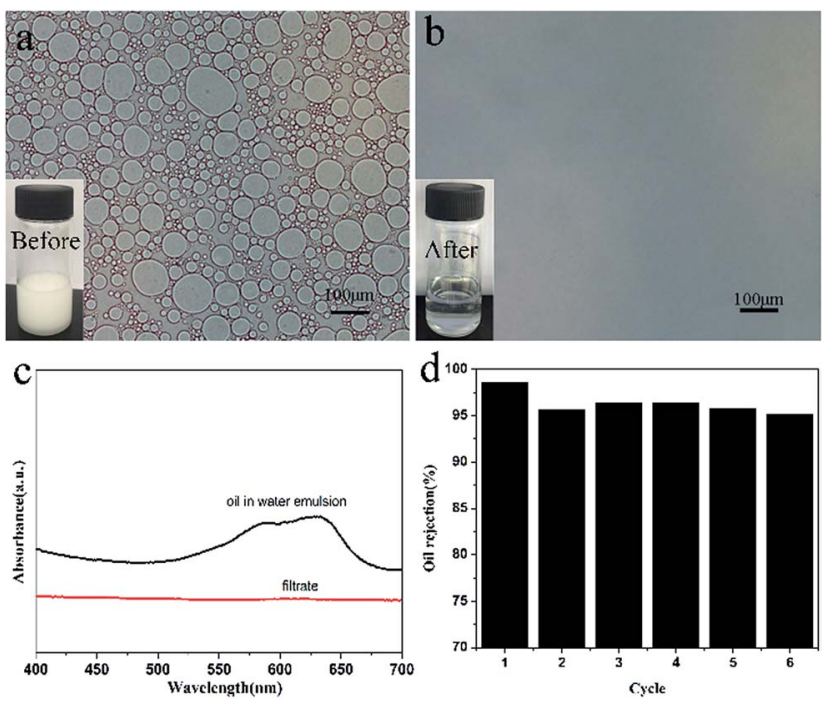

Fig. 6 (a) and (b) Photographs of surfactant-stabilized n-hexane-inwater emulsion before and after separation (scale bar: $100 \mu \mathrm{m}$ ). (c) UVVis spectrum of the oil in water emulsion before and after separation, (d) cyclic separation of the SCS-MPA to oil-to-water emulsion.

(Fig. 6c). This was comparable to reported rejection ratio of cellulose nanofiber aerogels $(98.6 \%)^{20}$ and that of cellulose membranes (99.6\%). ${ }^{40}$ And these results were illustrated the high separation efficiency of the wetted cellulose sponge. The used sponge could be reused after rinsing with D.I. water repeatedly until free of any oil-dissolved disperse dyes. The rejection ratio of the oil-in-water emulsion remained over $95 \%$ up to six reuse cycles (Fig. 6d).

\section{Conclusions}

In summary, a novel hydrophilic sponge with enhanced mechanical properties was prepared by silylation of cellulose fibrils and subsequent modification by thiol-ene click reaction. The sponge was successfully utilized for oil removal from both oil/water mixture and an oil-in-water emulsion. The thiol-ene modification endowed the sponge with superb hydrophilicity and underwater oleophobicity $\left(147^{\circ}\right)$. The sponge also boasted high porousity ( $>99 \%)$, excellent compressive strength (70 KPa) and decent stability and flexibility, allowing it to be repeatedly used. The separation efficiency was as high as $100 \%$ after 10 cycles for oil/water mixture and over $95 \%$ after 6 cycles for oil-inwater emulsions. It is worth noting that the cellulose sponge gained high wet strength and durability underwater from crosslinking by VTMO. These results indicated that the hydrophilic material has great potential in separating oil/water emulsion.

\section{Acknowledgements}

This work was financially supported by National Natural Science Foundation of China (No. 51403035), and the Fundamental Research Funds for the Central Universities (No. 16D110510). 


\section{References}

1 A. Venkateswara Rao, N. D. Hegde and H. Hirashima, J. Colloid Interface Sci., 2007, 305, 124-132.

2 S. Yun, H. Luo and Y. Gao, RSC Adv., 2014, 4, 4535-4542.

3 R. Zhang, L. Hu, S. Bao, R. Li, L. Gao, R. Li and Q. Chen, J. Mater. Chem. A, 2016, 4, 8412-8420.

4 Y. Meng, T. M. Young, P. Liu, C. I. Contescu, B. Huang and S. Wang, Cellulose, 2014, 22, 435-447.

5 H. Hu, Z. Zhao, W. Wan, Y. Gogotsi and J. Qiu, Adv. Mater., 2013, 25, 2219-2223.

6 S. Kabiri, D. N. H. Tran, T. Altalhi and D. Losic, Carbon, 2014, 80, 523-533.

7 S. Yu and H. Tan, ACS Appl. Mater. Interfaces, 2015, 7, 67456753.

8 Q. F. Wei, R. R. Mather, A. F. Fotheringham and R. D. Yang, Mar. Pollut. Bull., 2003, 46, 780-783.

9 X. Zhang, L. Zhang, K. Liu and L. Jiang, Adv. Funct. Mater., 2013, 23, 2881-2886.

10 C. F. Wang, H. C. Huang and L. T. Chen, Sci. Rep., 2015, 5, 14294.

11 D. Klemm, B. Heublein, H. P. Fink and A. Bohn, Angew. Chem., Int. Ed., 2005, 44, 3358-3393.

12 J. Feng, S. T. Nguyen, Z. Fan and H. M. Duong, Chem. Eng. J., 2015, 270, 168-175.

13 T. Zhai, Q. Zheng, Z. Cai, H. Xia and S. Gong, Carbohydr. Polym., 2016, 148, 300-308.

14 Z. Zhang, G. Sèbe, D. Rentsch, T. Zimmermann and P. Tingaut, Chem. Mater., 2014, 26, 2659-2668.

15 Q. Liao, X. Su, W. Zhu, W. Hua, Z. Qian, L. Liu and J. Yao, RSC Adv., 2016, 6, 63773-63781.

16 N. T. Cervin, C. Aulin, P. T. Larsson and L. Wågberg, Cellulose, 2011, 19, 401-410.

17 F. Wang, S. Lei, M. Xue, J. Ou and W. Li, Langmuir, 2014, 30, 1281-1289.

18 C. Su, Y. Xu, W. Zhang, Y. Liu and J. Li, Appl. Surf. Sci., 2012, 258, 2319-2323.

19 W. Zhang, Y. Zhu, X. Liu, D. Wang, J. Li, L. Jiang and J. Jin, Angew. Chem., 2014, 53, 856-860.

20 Z. He, X. Zhang and W. Batchelor, RSC Adv., 2016, 6, 2143521438.
21 K. Rohrbach, Y. Li, H. Zhu, Z. Liu, J. Dai, J. Andreasen and L. Hu, Chem. Commun., 2014, 50, 13296-13299.

22 H. C. Kolb, M. G. Finn and K. B. Sharpless, Angew. Chem., Int. Ed., 2001, 40, 2004-2021.

23 A. B. Lowe, Polym. Chem., 2010, 1, 17-36.

24 P. Tingaut, R. Hauert and T. Zimmermann, J. Mater. Chem., 2011, 21, 16066.

25 Y. Wang, T. Heinze and K. Zhang, Nanoscale, 2016, 8, 648657.

26 J. Guo, W. Fang, A. Welle, W. Feng, I. Filpponen, O. J. Rojas and P. A. Levkin, ACS Appl. Mater. Interfaces, 2016, 8, 3411534122.

27 A. P. Rao, A. V. Rao and G. M. Pajonk, Appl. Surf. Sci., 2007, 253, 6032-6040.

28 H. Guo, B. N. Nguyen, L. S. McCorkle, B. Shonkwiler and M. A. B. Meador, J. Mater. Chem., 2009, 19, 9054.

29 T. Matias, C. Varino, H. C. de Sousa, M. E. M. Braga, A. Portugal, J. F. J. Coelho and L. Durães, J. Mater. Sci., 2016, 51, 6781-6792.

30 S. Smitha, P. Shajesh, P. Mukundan and K. G. K. Warrier, J. Sol-Gel Sci. Technol., 2007, 42, 157-163.

31 M. Zaharescu, A. Jitianu, A. Braileanu, J. Madarász and G. Pokol, J. Therm. Anal. Calorim., 2001, 64, 689-696.

32 H. Sehaqui, Q. Zhou and L. A. Berglund, Compos. Sci. Technol., 2011, 71, 1593-1599.

33 A. J. Svagan, M. A. S. A. Samir and L. A. Berglund, Adv. Mater., 2008, 20, 1263-1269.

34 H. Sai, R. Fu, L. Xing, J. Xiang, Z. Li, F. Li and T. Zhang, ACS Appl. Mater. Interfaces, 2015, 7, 7373-7381.

35 C. Aulin, J. Netrval, L. Wågberg and T. Lindström, Soft Matter, 2010, 6, 3298.

36 H. Huang, P. Chen, X. Zhang, Y. Lu and W. Zhan, Small, 2013, 9, 1397-1404.

37 X. Yang and E. D. Cranston, Chem. Mater., 2014, 26, 60166025.

38 Q. Fu, X. Wang, Y. Si, L. Liu, J. Yu and B. Ding, ACS Appl. Mater. Interfaces, 2016, 8, 11819-11829.

39 Y. Si, Q. Fu, X. Wang, J. Zhu, J. Yu, G. Sun and B. Ding, ACS Nano, 2015, 9, 3791-3799.

40 Y. Cao, N. Liu, W. Zhang, L. Feng and Y. Wei, ACS Appl. Mater. Interfaces, 2016, 8, 3333-3339. 